\title{
Biopolymer Sequencing
}

National Cancer Institute

\section{Source}

National Cancer Institute. Biopolymer Sequencing. NCI Thesaurus. Code C116155.

A process to identify and determine the primary structure of, and the order of constituents in a biopolymer. 